\title{
Image Browsing on a Large Display
}

\author{
Anna Hedman \\ Institutionen för systemteknik \\ Luleå tekniska universitet \\ SE-971 87 Luleå, Sweden \\ hedman@ltu.se
}

\begin{abstract}
We conducted a usability study on a 50" plasma display comparing three different browser alternatives (iconic, zoom-and-pan, and bifocal view) for an electronic bulletin board containing thumbnails of notes. For input device we used a $3 D$ mouse. We conducted an experiment where 10 subjects performed browsing tasks with each browser. We found that users performed significantly better with the iconic browser than with the zoom-and-pan-browser. There was no significant difference between the fisheye browser and any of the other two. The users preferred the iconic browser.
\end{abstract}

Keywords. Thumbnail browsing, large display, input device, user study, zooming user interface, fisheye interface.

\section{Introduction}

A computer screen can only view a limited amount of information at readable size at the same time. For this problem, there are two solutions: Either use a browsing technique, like for instance zoom, pan, scroll bars, or fisheye, or get more screen space.

Large displays are becoming a reality for an increasing number of users as prices go down. So far, browsing techniques have mainly been studied on regular desktop displays.

There are many usability issues to consider when display size differs radically from standard size of desktop displays. Freehand interaction with other input devices than the regular 2D desktop mouse, for instance, is a highly relevant part of interaction with large displays.

This study is mainly a comparison of browsing techniques, but we also discuss observations made regarding interaction with a large display and a 3D input device. The browser alternatives included in this study are: an iconic browser, a zoom-and-pan browser, and a fisheye browser [7]. Our iconic browser operates in a manner similar to the standard windowing system. Clicking a thumbnail replaces the bulletin board with a readable-sized version of the note. Our zoomand-pan browser was implemented by using the Piccolo toolkit [3] with modified zoom function. Finally, we implemented a type of fisheye browser called the "bifocal view" [13].

In order to determine which had the best performance, we conducted a usability study comparing the iconic, zoom-and-pan, and bifocalview browsers on a 50 " plasma display with a $3 \mathrm{D}$ mouse. Ten subjects completed our study. Each participant used each browser during the experiment. After a review of related work, we will describe the browsers and the study in detail. This will be followed by a discussion of the results and a summary with a discussion of future work.

\section{Related Work}

In their image-browser taxonomy, Plaisant, Carr, and Shneiderman [16] describe different types of tasks and how these benefit from different types of browser characteristics.

Several browser studies have been conducted, with various results. No single browser has been better overall than the others for all types of tasks.

In a study comparing scroll bars, dragging, a pop-up overview, and a pop-up overview containing a field-of-view indicator, Kaptelinin found that the interfaces with a pop-up overview were significantly faster than both the scroll-bar interface and the drag interface [12].

Beard and Walker [2] compared interfaces using scrollbars, zoom, and roam (pan) with and without overview, and found that all techniques gave better results with the overview, and scroll bars were significantly slower than the other two techniques.

A study by Hornbæk, Plaisant, and Bederson [11] comparing zooming interfaces with and without an overview found that subjects were faster without the overview, but preferred an interface that had one. 


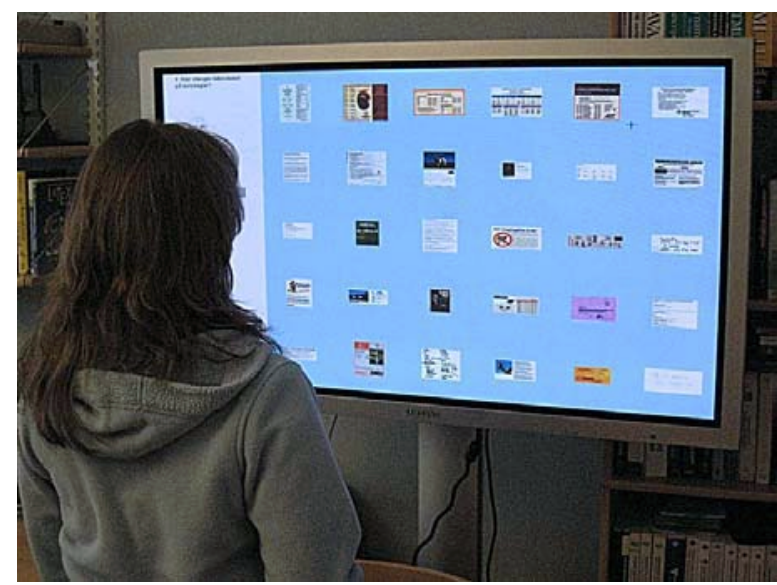

Figure 1. The test program on the 50" display

Donskoy and Kaptelinin [6] compared scrollbar, zoom, and fisheye interfaces with and without animation, and found that the lowest task completion times were achieved with the zooming interface, with animation as well as without. The fisheye turned out to be the slowest technique, and was also the only technique that was faster with animation than without. However, subjects preferred animation in general.

In a study into how browsers affect the readability of electronic documents, Hornbæk and Frøkjær [10] compared linear, fisheye, and overview-plus-detail interfaces, finding that participants performed faster with the fisheye interface on essays but received higher grades with the overview-plus-detail interface.

In a study on the efficiency of fisheye browsers, Schaffer, et al. [18] compared fisheye and zoom-and-replace interfaces on node-link diagrams representing a telephone network. For this task, the fisheye view was significantly faster than the zoom-and-replace.

Combs and Bederson [4] compared four browsers for photographs: One thumbnail browser featuring a horizontal scrollbar, a zoomand-pan browser, and two 3D browsers. Participants performed better with both $2 \mathrm{D}$ browsers than with the $3 \mathrm{D}$ browsers. Furthermore, they also made fewer incorrect selections with 2D browsers, and rated them higher.

Gutwin and Fedak [8] made a comparison on small screens, finding that a fisheye interface was useful for web navigation tasks, and a twolevel zoom for a monitoring task, while panning slowed down users.

In research on large displays several usability issues has already been covered. Robertson et al. [17] have addressed several problems with proposed software solutions. An extensive overview on large display research is given by Czerwinski et al. [5].

In a summary of observations from a large display project, Swaminathan and Sato [19] identified characteristics of the regular 2D mouse, such as the fact that it can cover the entire width of an SVGA display in one single hand movement with fairly high accuracy, which might not be possible with a large display with high resolution.

MacKenzie and Jusoh found that users perform better with a regular mouse than with any of their two examined input devices for remote pointing [14]. However, a regular desktop mouse cannot be used in situations where there is no support from a table or any other stable surface. Clearly, there is a need for other types of input devices.

Myers at al. investigated performance of laser pointers and other devices for interacting at a distance, and found that laser pointer allows the user to interact at a distance, but with low accuracy than with a regular mouse. They also found that tapping immediately on the board is efficient, but this technique limits the distance between the user and the display [15].

With this study, we wish to combine browsing studies with large display usage and freehand interaction.

\section{The Usability Experiment}

In order to determine which type of browser works best for a set of images on a large screen, and investigate behavior with a large display and a different input device than a $2 \mathrm{D}$ mouse, we conducted an experiment to compare three browsing techniques: iconic, zoom-and-pan, and a fisheye of the type called a "bifocal view". We recruited 10 participants, who all performed a set of tasks with each of the different browsers in a within-subjects experiment design. For each participant, we measured task completion times and number of errors. These became our dependant variables with browser type and task set as independent variables. We also asked the participants to rate each technique on a scale from 1 (bad) to 7 (good), and collected their comments on each browser.

\subsection{Equipment}

The browsers were implemented in Java on a $3 \mathrm{GHz}$ Pentium 4 running Windows XP. The system had a 50" plasma display with a $1024 \times 768$ 
resolution (Fig. 1). For input device we used a Wanda (Fig. 2) with a Flock of Birds and an Extended-Range Transmitter from Ascension Tech [1]. The test program divided the display vertically with a task panel covering the left fifth. The questions were displayed and answered in this panel. The rest of the display was used for displaying notes on a bulletin board that used one of three browser types: iconic, zoom-and-pan, or bifocal. All answers and task times were logged by the test program. The board was restored to the original view with all notes automatically before each task, but there was also a restore button in the bottom of the left corner that allowed users to return to the original overview at anytime. For all techniques, pressing the right mouse button would clutch the cursor and allow the user to adjust the position of the hand and arm. To avoid fatigue the user was allowed to rest between the tasks.

\subsection{The Browsers}

The iconic browser worked almost the same as a standard iconic desktop. When an icon was clicked with the left button, the view of the entire board was replaced by a larger version of the selected note. Minimizing the note was done by clicking again.

The zoom-and-pan browser was implemented using Piccolo [3] with modifications for movements in three directions with the Wanda. Initially, it displayed all notes on the screen. Zooming in or out was done by pressing the middle mouse button and moving the Wanda towards or away from the screen. The view could be panned in any direction in the $x-y-p l a n e$ by moving the

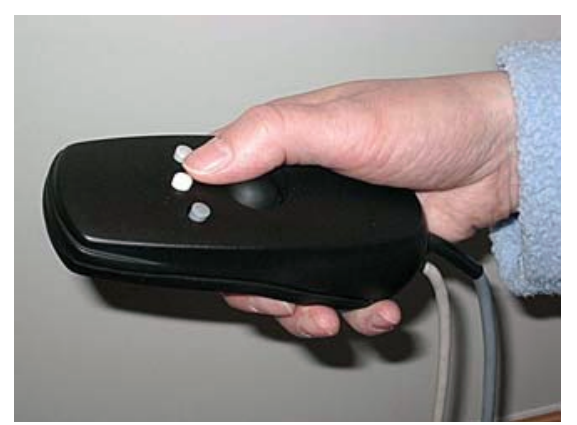

Figure 2. The Wanda

Wanda in the corresponding direction with the left button pressed.

The bifocal-view [13] browser (Fig. 3) consists of $3 \times 3$ regions where the middle section is a flat magnifying glass that can be dragged in any direction to view any part of the bulletin

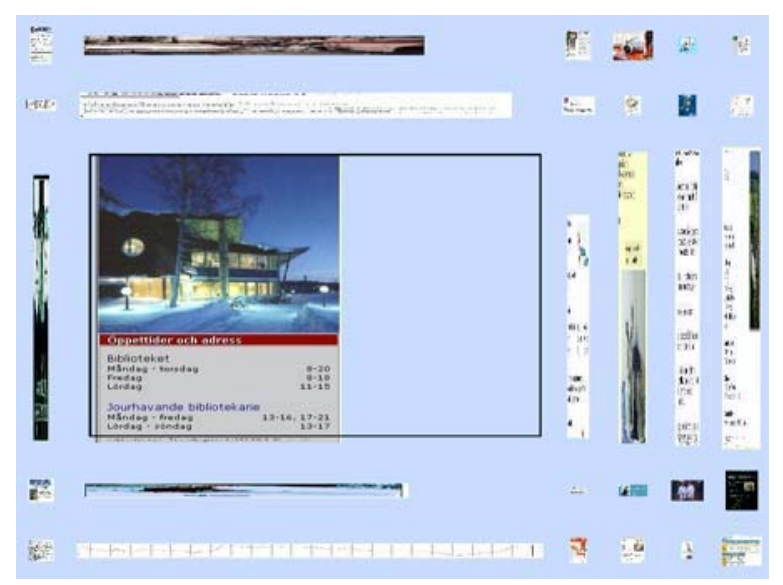

Figure 3. The bifocal display

board. The other regions consisted of the distorted surroundings. In the bifocal browser it was possible to select a note outside the focus area and have the focus moved instantly to that note. The glass was moved according to an acceleration function that enabled small movements at low rates and large movements at higher rates.

\subsection{Tasks}

We conducted a within subjects test where each of the participants used all three techniques, iconic, bifocal, and zoom-and-pan. All browsers were presented with a set of eight tasks with a corresponding set of 30 notes. To avoid a learning effect we used three different sets of notes and an extra set for practicing the techniques before each actual test. All sets of notes were fairly equal regarding content, size and color of the notes. Notes containing answers were distributed throughout the bulletin board. The order of browsers and sets was randomly assigned for each participant and counterbalanced.

The goal of each task was to retrieve certain requested information, such as closing hours for the library on a certain day, or the phone number for a given person, by browsing the bulletin board. Each question was presented with four alternative answers and an alternative for skipping the question in case the participant felt uncomfortable with a task.

\subsection{Test Participants}

Of the 10 participants whose data was analyzed, 8 were men and 2 were women. All participants were experienced with computers and used them frequently. The predominant operating system was some version of Microsoft Windows. 
A majority used computers for office work and surfing the Internet.

\subsection{Hypotheses}

We expected the following results from this experiment:

- The iconic browser would be more efficient than both the zoom-and-pan and the bifocal browser.

- The zoom-and-pan browser would be more efficient than the bifocal browser.

- Subjective preference would be for the iconic browser, and zoom-and-pan would be preferred over the bifocal view.

A comparison of these three techniques was previously made on a regular 19" screen [9]. This study showed a significant difference between the iconic browser and the other two browsers. There was no significant difference between the zoom-and-pan browser and the bifocal browser. Before conducting the test on a 50" display, we made some adjustments for the 3D input device and some minor changes based on user comments and observations from that study. In spite of these changes, we still expected the iconic to be the most efficient and also most appreciated by the users because of familiarity. The iconic browser also produces a fast response, which we believe compensates for not having the overview available all the time.

Zooming with the Wanda was done towards and away from the screen, which we believed would feel natural and intuitive to the user. Animated zoom gives a good overview of the notes and maintains a smooth transition, which should allow incidental learning of neighboring notes. However, animation time in a zoom-and-pan browser is significant compared to the pop-up time in an iconic browser, and not having the overview available can cause the user to become lost in the details. In addition, learning a new and unfamiliar technique might require more practice over a longer time. For these three reasons, we expected this technique to be slower than the iconic browser.

We believed that the bifocal browser would be the slowest, partly because of the distortion that made the surroundings move when the focus area was dragged. Furthermore, moving the focus area from one edge of the board to the other required either a fast move, which made it impossible to see the contents of the notes, or sev- eral slower hand movements, which was fatiguing and time consuming.

\subsection{Results}

We performed a multifactor ANOVA with task times as the dependent variable. Independent variables were: browsing technique, task set, and browser order. There was a significant effect for browsing technique $(F=3.57, p=0.0298)$. No significant effect was found for task set or browser order. We then performed range tests with a 95\% Least Significant Difference (LSD), for browsing technique. The test showed the iconic browser to be significantly faster than the zoom-and-pan browser, but not the bifocal-view browser (Fig. 3). No significant difference was found between the bifocal browser and the zoom-and-pan browser. Errors were not a significant factor. On 240 tasks ( $10 \times 3 \times 8), 6$ were excluded from the analysis, five due to various

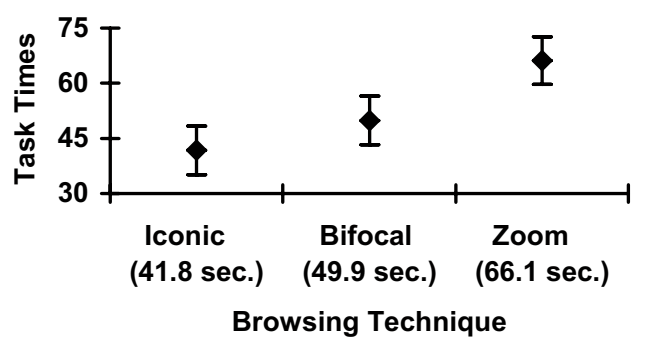

Figure 2. Browser means with $\mathbf{9 5 \%}$ intervals

user related problems, and one due to a technical problem. On the 236 tasks that remained, there were only two incorrect answers.

\subsection{Subjective Satisfaction}

The participants were asked to rate each technique on a scale from one (bad), to seven (good). The iconic browser received higher ratings (mean 5.6) than the other techniques. Participants' ratings for the iconic browser were either 5 or 6 (Fig. 5), while their ratings for the zoomand-pan browser seemed to be a little lower (mean 4.4) and spread throughout the range. The bifocal-browser ratings (mean 2.8) were never higher than 4.

\section{Discussion}

Not all of our hypotheses were upheld. While the iconic browser was faster than the zoom-andpan browser, there was no significant difference between the iconic and the bifocal browsers, or 


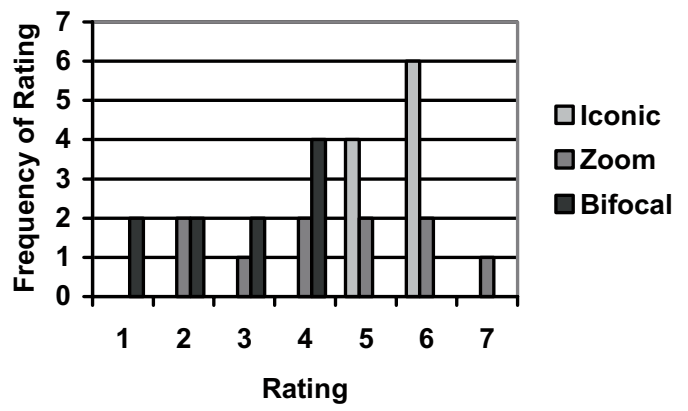

Figure 5. Distribution of user preference by browser type.

between the bifocal and the zoom-and-pan browsers.

An interesting observation by Swaminathan and Sato [19] on readability on large displays is that size actually seems to add more than resolution does, which we also noticed. With the original overview displayed, some of our participants tried to browse the board with their eyes instead of using the browsing technique. This was particularly obvious with the iconic browser, which was the only browser that required the user to frequently return to the overview. Although they could not actually read the contents without enlarging the notes, some participants tried to identify the target note, and to exclude notes that they believed were unlikely to hold the requested information without using the browsing technique. A problem with this strategy is that if the target note is among the notes that the user excludes in the search, the note will not be found until the user starts to systematically go through all notes. With the zoom-and-pan or the bifocal browser, it would be possible to discover such a mistake by accidentally viewing the target note when moving the focus area by dragging. Most users appreciated using the iconic browser, and expressed familiarity as a reason for why it felt comfortable. However, some users did not appreciate being forced to constantly switch between detail and overview.

As with the iconic browser, the zoom-and-pan browser did not allow viewing details and overview at the same time. With this technique the user could browse by panning without constantly switching back and forth between two views. Several users zoomed in to a level where they could see 4-6 notes on the screen, and then panned the board. The major drawback with not returning to the overview was that users did not always realize that they had not covered all notes. Three users stated that they preferred this technique to the iconic browser. A couple of us- ers expressed a wish for being able to continue panning even when the cursor hade reached the edge of the display. They felt that panning should be allowed for as long as their arm could reach in one move.

The bifocal view was the least popular technique among users. The focus area was moved either by dragging it or by clicking on a note in the distorted surroundings. Clicking seemed to be preferred over dragging. To make an attempt at trying to identify a possible target note, the user returned to the undistorted overview first, and then clicked on a note. To browse all notes, the user clicked on each object outside the magnifying area, without returning to the undistorted overview first. Dragging the glass was mainly used for adjusting the magnified area over a note in order to find relevant information. The two major drawbacks with this technique were the moving surroundings, and the inefficient and somewhat fatiguing drag function. We believe that a possible reason for why there was no significant difference between the iconic and the bifocal browser was that it the click function in the bifocal browser made it possible to use it almost as an iconic browser. However, with the bifocal browser distorted notes were hard to recognize, and the focus area were limited since space was sacrificed for the surrounding context.

\section{Conclusions and Future Work}

We have tested three possible interfaces for an electronic bulletin board: an iconic browser, a zoom-and-pan browser, and a bifocal-view browser. Participants were faster with the iconic browser than with the zoom-and-pan browser, but there was no significant difference between the iconic browser and the bifocal browser, or between the bifocal browser and the zoom-andpan browser. There was no difference in error rate among the browsers. The statistical analysis would have benefited from a greater number of participants. However, the observations made during this experiment seem to support the result and should be of interest for future studies.

It was our impression that clicking is better for data represented by several single objects, while techniques like bifocal and zoom are better for "continuous data". This theory is supported by the fact that the iconic browser received the highest ratings. Three participants claimed to like the zoom-and-pan even better than the iconic browser, but none of them performed faster with the zoom-and-pan. In a follow-up to this study, 
we intend to compare these techniques using maps instead of small representations of notes. We also wish to further investigate characteristics and usability of the system used in this study.

\section{Acknowledgements}

Thoughts of gratitude go to Professor Håkan Alm for good support and guidance, and $\mathrm{Dr}$ David A. Carr, who unfortunately is no longer with us. The equipment used in this study was financed by The European Commission's Goal 1 structural fund program for northern Norrland (vITal project, DNo. 304-6343-2002). Thanks also to Robert Lundqvist for statistical advice. Finally, thanks to the test participants without whom this work would have been impossible.

\section{References}

[1] Ascension Tech, http://www.ascensiontech.com

[2] Beard, D., Walker II, J., Navigational techniques to improve the display of large twodimensional spaces, Behaviour and Information Tech., 9(6), Nov. 1990, 451-466.

[3] Bederson, B. B., Grosjean, J., \& Meyer, J., Toolkit Design for Interactive Structured Graphics, IEEE Transactions on Software Engineering, 30 (8), 2004, 535-546.

[4] Combs, T., Bederson, B. B., Does zooming improve image browsing? Proc. of the $A C M$ Conference on Digital Libraries, 11-14 Aug 1999, Berkeley, CA, 130-137.

[5] Czerwinski, M., Robertson, G.G., Meyers, B., Smith, G., Robbins, D., Tan, D., Large display research overview, Proceedings of ACM's CHI 2006, 2006, 69-74.

[6] Donskoy, M., Kaptelinin, V., Window navigation with and without animation: a comparison of scroll bars, zoom, and fisheye view, Extended Abstracts of Human Factors in Computing Systems (CHI '97), Atlanta, GA, 22-27 Mar. 1997, 279-280.

[7] Furnas, G. W., Generalized fisheye views, Proceedings of the SIGCHI Conference on Human Factors in Computing Systems, Boston, MA, 13-17 Apr. 1986, 16-23.

[8] Gutwin, C., Fedak, C., Interacting with Big Interfaces on Small Screens: a Comparison of Fisheye, Zoom, and Panning Techniques, Proceedings of Graphics Interface, 2004, 19-26.

[9] Hedman, A., Carr, D., Nässla, H., Browsing Thumbnails: A Comparison of Three Tech- niques, Proceedings of the 26th International Conference on Information Technology Interfaces, 7-10 June 2004, 353-360.

[10] Hornbæk, K., Frøkjær, E., Reading patterns and usability in visualizations of electronic documents, ACM Transactions on Copmuter-Human Interaction (TOCHI), vol 10(2), June 2003, 119-149.

[11] Hornbæk, K., Bederson, B. B., Plaisant, C., Navigation patterns and usability of zoomable user interfaces with and without an overview, ACM Trans on Computer-Human Interaction, 9(4), Dec. 2002, 362-389.

[12] Kaptelinin, V., A comparison of four window navigation techniques in a $2 \mathrm{D}$ browsing task, Conference Companion on Human Factors in Computing Systems (CHI'95), Denver, CO, 7-11 May 1995, 282-283.

[13] Leung, Y. K., Apperley, M. D., A review and taxonomy of distortion-oriented presentation techniques, ACM Transactions on Computer-Human Interaction, 1(2), June 1994, 126-160.

[14] MacKenzie, I., Jusoh, D., An evaluation of two input devices for remote pointing, Eighth IFIP Working Conference on Engineering for Human-Computer Interaction, 2001, 235-249.

[15] Myers, B., Bhatnagar, R., Nichols, J., Peck, C., Kong, D., Miller, R., Long, A., Interacting at a distance: Measuring the performance of laser pointers an other devices, Proceedings of the ACM Conference on Human Factors in Computing Systems (CHI 2002,, 2002, 33-40.

[16] Plaisant, C., Carr, D., Shneiderman, B., Image browser taxonomy and guidelines for designers, IEEE Software, 12(2), Mar. 1995, 21-32.

[17] Robertson, G.G., Czerwinski, M., Baudisch, P., Meyers, B., Robbins, D., Smith, G., Tan, D., Large Display user experience, IEEE $C G \& A$ special issue on large displays, 25(4), 2005, 44-51.

[18] Schaffer, D., Duo, Z., Greenberg, S., Bartram, L., Dill, J., Dubs, S., Roseman, M., Navigating hierarchically clustered networks through fisheye and full-zoom methods, ACM Trans. on Computer-Human Interaction, 3(2), Mar. 1996, 162-188.

[19] Swaminathan, K., Sato, K. Interaction Design for large displays, ACM's Interactions (4), 1997, 15-24. 